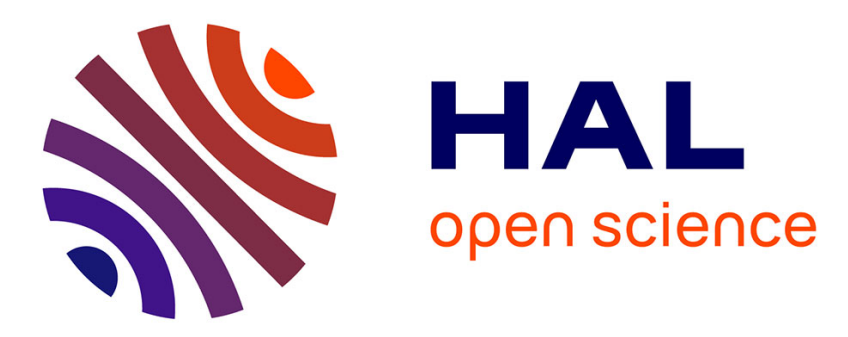

\title{
Note préliminaire sur le site protohistorique de Punta di Casteddu (Sartène, Corse-du-Sud)
}

\author{
Kewin Peche-Quilichini, Florian Soula, Florent Châteauneuf
}

\section{To cite this version:}

Kewin Peche-Quilichini, Florian Soula, Florent Châteauneuf. Note préliminaire sur le site protohistorique de Punta di Casteddu (Sartène, Corse-du-Sud). Gallia Préhistoire - Préhistoire de la France dans son contexte européen, 2016, 56, pp.198-212. 10.4000/galliap.390 . hal-01765919

\section{HAL Id: hal-01765919 \\ https://hal.science/hal-01765919}

Submitted on 2 Jan 2020

HAL is a multi-disciplinary open access archive for the deposit and dissemination of scientific research documents, whether they are published or not. The documents may come from teaching and research institutions in France or abroad, or from public or private research centers.
L'archive ouverte pluridisciplinaire HAL, est destinée au dépôt et à la diffusion de documents scientifiques de niveau recherche, publiés ou non, émanant des établissements d'enseignement et de recherche français ou étrangers, des laboratoires publics ou privés.

\section{(ㅇ)(1) $\$$}

Distributed under a Creative Commons Attribution - NonCommercial - NoDerivatives $\mid 4.0$ 


\title{
Note préliminaire sur le site protohistorique de Punta di Casteddu (Sartène, Corse-du-Sud)
}

\author{
Kewin Peche-Quilichini ${ }^{*}$, Florian Soula ${ }^{* *}$, Florent Châteauneuf ${ }^{* *}$
}

Moтs clés. Corse méridionale, Sartenais, Bronze final, premier âge du Fer, nécropole, crémation, habitat, territoire, espaces culturels, céramique.

Résumé. Le site protohistorique de Punta di Casteddu se situe dans le sud-ouest de la Corse, dans la région du Sartenais. Découvert il y a une vingtaine d'années, il a récemment été réexaminé à l'occasion d'une prospection pédestre menée dans le cadre du programme de recherche "Cauria, site mégalithique de Stazzona - I Stantari", dirigé par A. D’Anna. Le site est occupé et utilisé à partir de l'étape médiane du Bronze final et jusque vers le milieu du premier âge du Fer, soit la période de loin la mieux représentée pour les périodes pré- et protohistoriques dans la microrégion. II inclut un secteur d'activités domestiques comprenant une à deux habitation(s) et plusieurs groupes d'abris-sous-roche. Parmi ces derniers, le secteur occidental semble avoir connu une utilisation intensive marquée par une importante quantité de vestiges matériels superficiels, notamment céramiques. Ces vaisselles sont caractéristiques de la transition Bronze/Fer de cette partie de l'île qui connaît probablement un important essor démographique et une remarquable unité culturelle dès la fin du I/ ${ }^{e}$ millénaire avant J.-C. De manière encore hypothétique, on envisage ici une utilisation funéraire de la plupart de ces cavités, selon un postulat introduisant pour la première fois l'éventualité de l'incinération, qui serait arrivée dans lîle depuis les rives de l'Italie nord-occidentale dès le début du Bronze final, voire avant, et qui expliquerait en partie la carence de contextes sépulcraux pour cette phase de la Protohistoire de la Corse.
Kerwords. Southern Corsica, Sartenais, Late Bronze Age, Early Iron Age, funerary site, cremation, settlement, territory, culture sphere, ceramics.

Abstract. The protohistoric site of Punta di Casteddu is located in south-western Corsica, in the Sartenais region. It was discovered some twenty years ago and has recently been reassessed as part of an archaeological fieldwalking survey carried out within the research project "Cauria, the megalithic site of Stazzona I Stantari" directed by André D'Anna. The site was occupied and used from the middle of the Late Bronze Age to the middle of the Early Iron Age, i.e. the pre- and protohistoric periods by far the best represented in this micro-region. The site contains evidence of domestic activity including one or two houses and several clusters of rock cavities or shelters. Among the latter, the western group appears to have been extensively used, as shown by large quantities of material recovered from the surface, especially pottery. This pottery is typologically and technologically typical of the Bronze Age-Iron Age transition in this part of the island that probably saw a significant level of demographic growth and a remarkable degree of cultural unity from the end of the $2^{\text {nd }}$ millennium $B C$ onwards. As a working hypothesis we interpret most rock cavities as having had a funerary use, suggesting for the first time that cremation may have taken place there, introduced to the island from north-western Italy at the beginning of the Late Bronze Age if not earlier. This would partly explain the absence of funerary contexts in this phase of Corsican protohistory.

*INRAP Méditerranée, laboratoire ASM - UMR 5140, 390 avenue de Pérols - F-34970 Lattes, baiucheddu@ gmx.fr

** post-doctorants, UMR 7269 - LAMPEA, Aix-Marseille Université, CNRS, Ministère de la culture et de la communication, 5 rue du Château de l'Horloge, BP 647, F-13094 Aix-en-Provence cedex 2, sflo1981@gmail.com / florent.chateauneuf@hotmail.fr 


\section{INTRODUCTION}

\section{CONTEXTE DE LA DÉCOUVERTE ET ÉTAT DES CONNAISSANCES}

C'est à l'occasion de prospections pédestres réalisées dans le cadre du programme de recherche "Cauria, site mégalithique de Stazzona - I Stantari » (D’Anna et al., 2006a) que la zone de la Punta di Casteddu ${ }^{1}$, située immédiatement à l'est du plateau de Cauria, dans le sud-ouest de la Corse, a fait l'objet de nouvelles explorations. Son toponyme ayant d'emblée attiré l'attention ${ }^{2}$, les recherches bibliographiques ont permis de relier à ce site la découverte ancienne de quelques tessons de céramique modelée de chronologie pré- et/ou protohistorique (Codaccioni, 2000, p. 155-156 ; Leandri et al., 1996, p. 30-31). La première personne à y signaler des vestiges est cependant P. Nebbia, conservateur du Musée départemental de Sartène, en 1994, qui y mentionne également la présence d'abrissous-roche peu accessibles sur le massif escarpé et couvert d'une végétation dense. Depuis 1994, la notice est simplement reprise, jusqu'à la redécouverte et l'étude préliminaire du site aux printemps 2009 puis 2010 .

\section{CONTEXTE ENVIRONNEMENTAL}

Située sur la commune de Sartène (Corse-du-Sud), la Punta di Casteddu est un sommet granitique accidenté, culminant aux environs de $228 \mathrm{~m}$ d'altitude (fig. 1). Enclavée entre le ruisseau de Salcinaja au nord et la vallée de l'Ortolu, à l'est et au sud, qu'elle domine presque entièrement, la pointe rocheuse présente un accès relativement aisé par l'est, par le sommet secondaire de Salcinaja, et un accès abrupt à l'ouest permettant d'arriver directement sur le petit plateau medio-sommital à partir de l'enclave de Petra Mattaja.

La Punta di Casteddu est un relief divisé en trois sommets culminant à 228, 160 et $166 \mathrm{~m}$, séparés par un petit plateau. Le sommet granitique principal, au nord, appartient à un faciès de monzogranites à grains moyens à fins traversé par des lames d'aplite et des filons de lamprophire qui composent une partie du massif voisin du Grecu (fig. 1, 2.a). Ce massif est entaillé dans un axe nord-ouest/sud-est par le fleuve de l'Ortolu, dans un axe est-ouest par le ruisseau de Salcinaja et dans un axe nord-sud par le ruisseau de Petra Mattaja. L'érosion et l'action tectonique ont provoqué des chutes d'énormes blocs sur ses pentes très raides, particulièrement sur les faces orientale et occidentale. Ces zones d'éboulis sont particulièrement propices à l'aménagement d'abris-sous-roche et de taffoni.

Depuis le sommet principal, qui domine le secteur occidental d'abris-sous-roche et le plateau (fig. 2, c) dont il sera question ici, l'observateur domine plusieurs écosystèmes : la

1. Littéralement, la « Pointe du Château », probablement en raison de la forme de son escarpement et de celle du rocher qui la surplombe, pouvant être assimilée à un donjon naturel.

2. Dans le sud de la Corse, la toponymie préfixée en CASTID/ ED (Castidetta, Castiddacciu, Castiddare, Castiglioni, Casteddu, Castedducciu) renvoie fréquemment à des lieux où sont implantées des fortifications protohistoriques. vallée de l'Ortolu, à l'est et au sud, constitue un grand axe naturel reliant la plage de Murtoli aux reliefs sartenais et par extension aux voies de communication entre l'Alta Rocca, le Sartenais et la pointe méridionale de l'île (Soula, 2012a, 2012b) (fig. 2, d-e). En direction du sud-ouest, la Punta di Casteddu domine l'écosystème s'étendant de Bocca di a Pila au plateau de Cauria, notamment enclavé entre la Punta di u Grecu et la Punta di a Villa (fig. 2, e). L'ensemble du plateau de Cauria et de ses environs constitue un sous-système environnemental très particulier qui marque une rupture très nette des reliefs entre la vallée de l'Ortolu et la vallée de la Navara. Il est séparé de la Punta di Casteddu par le massif de Tiresa - Petra Nera. Le col de Bocca di a Pila marque un lien naturel privilégié entre la Punta di Casteddu et le plateau de Cauria (fig. 2, e ; D'Anna et al., 2006a, 2006b, 2007a, 2007b).

\section{LES STRUCTURES IDENTIFIÉES}

La redécouverte du site a permis d'identifier deux zones documentées par de nombreux indices d'anthropisation, ainsi que deux groupements d'abris-sous-roche. Le matériel collecté durant les prospections se compose de 858 pièces, très majoritairement céramiques ${ }^{3}$. Comme on le verra par la suite, la grande majorité d'entre elles est constituée d'éléments allant dans le sens d'une attribution chronologique du site au Bronze final (1200-850 av. J.-C.) et au premier âge du Fer (850-550 av. J.-C.).

\section{L'HABITAT}

Le plateau central de la Punta di Casteddu se présente sous la forme d'une terrasse vaste de 1 à 2 hectares, légèrement en pente en direction du sud-ouest. Il constitue une plate-forme d'accès au gisement depuis les pentes occidentales (fig. 2, c). De nombreux tessons jonchent un maquis bas et faiblement arboré. Ce plateau constitue probablement le plus vaste secteur d'habitat de la Punta di Casteddu car il est le plus ouvert. Des tronçons de terrassements en gros blocs, probablement protohistoriques, ont été observés en bordure occidentale de ce plateau (fig. 3, b). Ils marquent vraisemblablement des divisions internes de l'espace ainsi que de très probables sections de consolidation des terrains, légèrement en pente et donc sujets à une érosion naturelle vers les bassins secondaires alimentant l'Ortolu. En contrebas de la pointe septentrionale, une ou deux structure(s) assimilable(s) à des habitations de type Cuciurpula (Peche-Quilichini et al., 2012) ont été identifiées (structures 1 et 2). Il s'agit d'agencements elliptiques de gros blocs de roche locale organisés en deux files convexes et symétriques s'appuyant contre un contrefort rocheux (fig. 3, c). Quelques éléments céramiques et une molette sont associés à ces structures dont l'architecture renvoie indéniablement au Bronze final et/ou au premier âge du Fer, et dont les avancées récentes de l'archéologie insulaire ont permis de documenter de façon assez précise le fonctionnement et l'évolution chronologique (PecheQuilichini et al., 2012, 2013, 2014a, 2014b, 2016, à paraître 1).

3. On compte également quelques meules (granit leucocrate) et molettes (microgranites et roches filoniennes). 

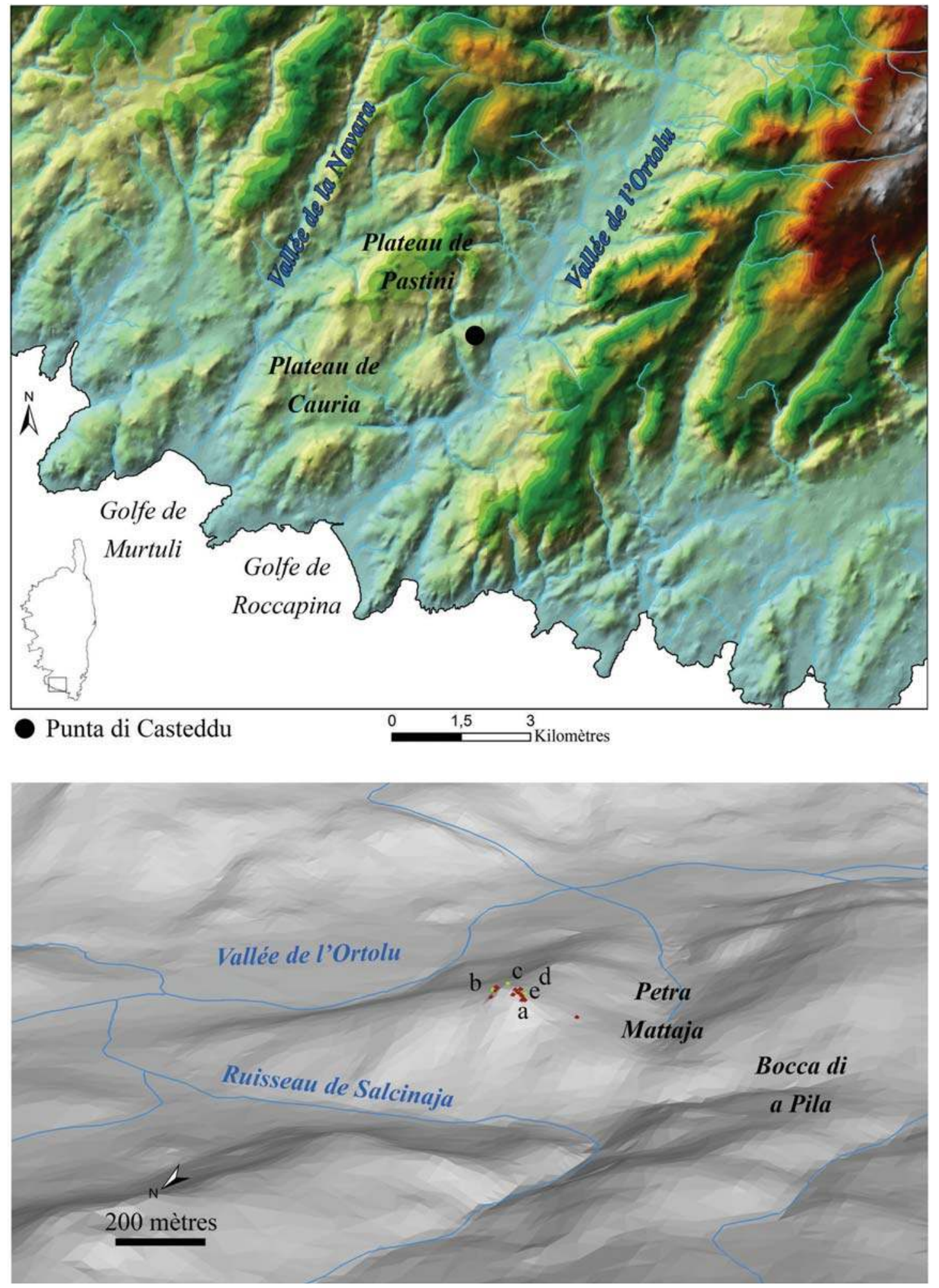

Fig. 1 - Carte de la région sud-sartenaise et vue tridimensionnelle de la Punta di Casteddu et de son contexte proche(DAO F. Soula, LAMPEA).

Map of the southern Sartenais region and three-dimensional view of the site of Punta di Casteddu in its local context (graphics: F. Soula, LAMPEA). 

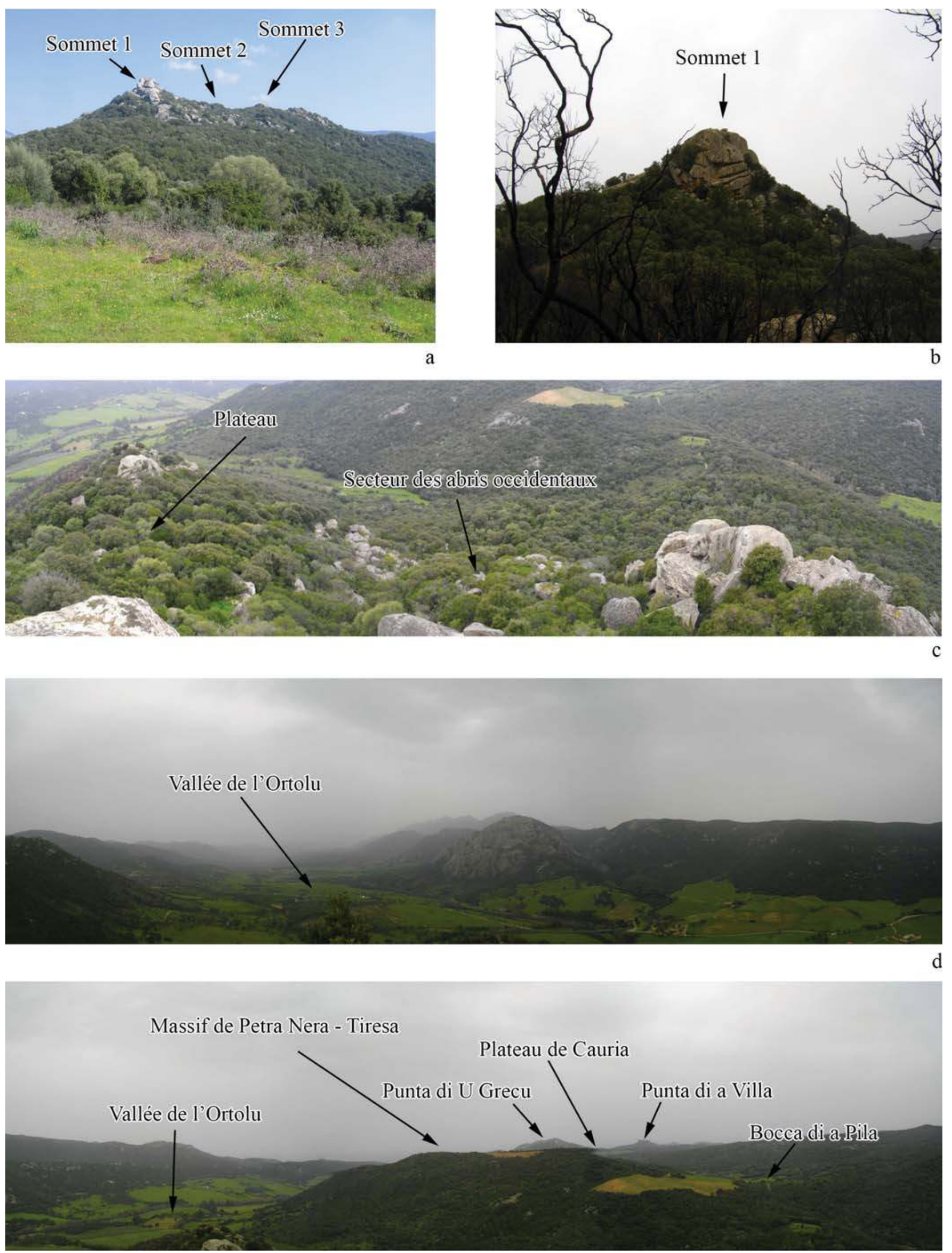
4 Fig. 2 - Vues du site de Punta de Casteddu (a-b) et depuis son sommet (c-e) (photos F. Soula, LAMPEA).

Views of the site of Punta de Casteddu (a-b) and from its summit (c-e) (photographs: F. Soula, LAMPEA).
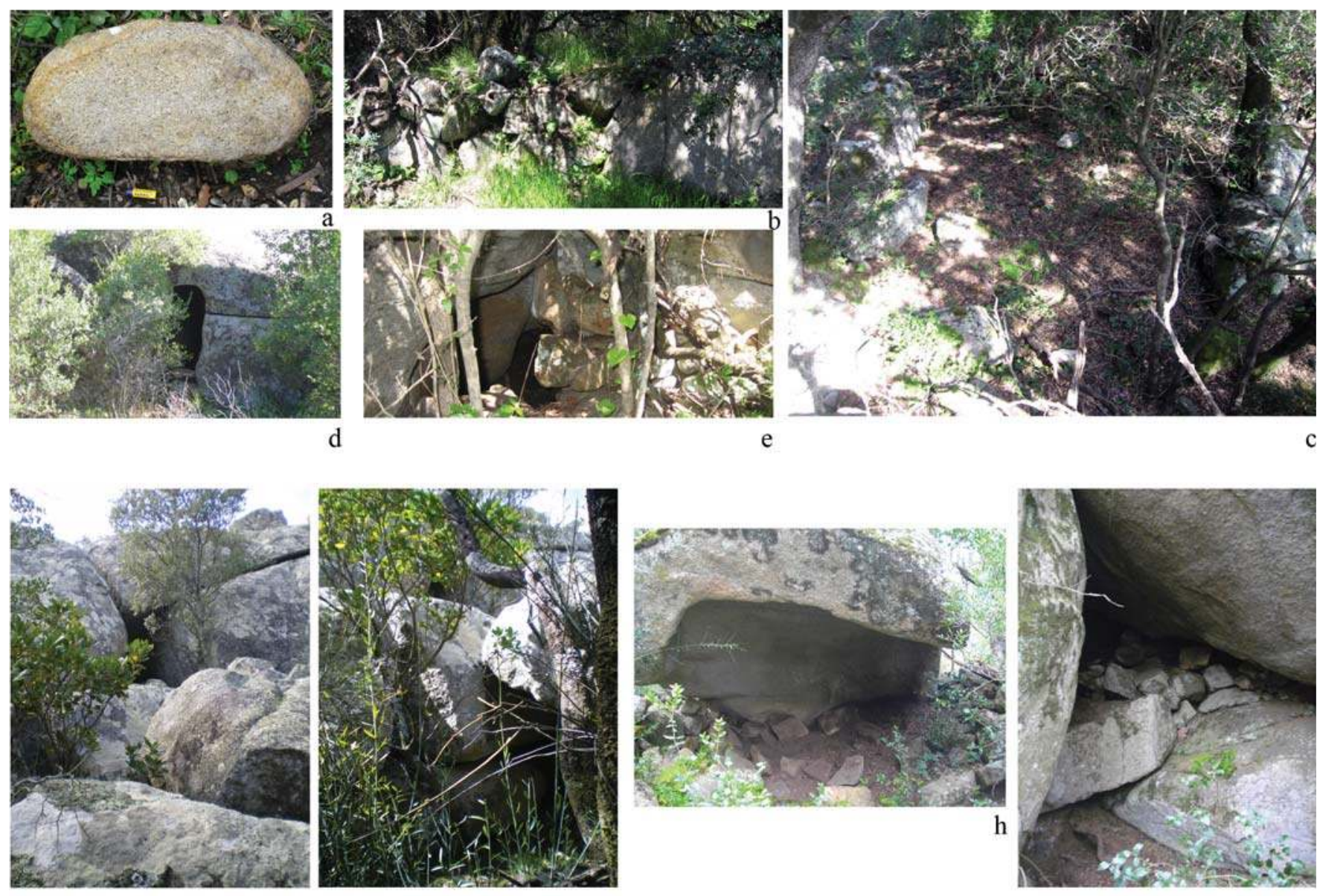

$\mathrm{f}$

Fig. 3 - Mobilier de mouture (a), terrassement (b), habitation (c), abris-sous-roche (d-i) (photos F. Soula, LAMPEA).

Grinding equipment (a), terracing (b), dwelling (c), rock cavities or shelters (d-i) (photographs: F. Soula, LAMPEA).

Enfin, l'exploration du secteur oriental du massif, non loin de la structure 1, a livré une dizaine de meules et quelques tessons épars témoignant de l'existence d'une seconde zone d'habitat et/ou d'activité de mouture. Ces éléments sont directement reliés à plusieurs abris-sous-roche dont certains ont vraisemblablement fonctionné de pair avec l'espace domestique mentionné ci-avant (fig. 3, h).

\section{LES ABRIS-SOUS-ROCHE}

Un nombre important d'abris-sous-roche a été identifié sur le site. L'utilisation d'une bonne part d'entre eux renvoie probablement à des fonctionnalités non directement domestiques car ils sont fréquemment exigus et difficiles d'accès. La totalité des cavités du secteur occidental (fig. 2, c, et 3, d-g) peut être attribuée à cette catégorie. Sur le secteur oriental, les abris sont généralement plus vastes, ouverts et plus aisément accessibles (fig. 3, h). Cette opposition typologique entre les cavités des deux secteurs est hypothétiquement corroborée par la présence d'un grand nombre d'éléments de mouture/broyage dans le secteur oriental, dans les abris où à proximité immédiate, alors que ceux du secteur occidental ne livrent que des éléments de vaisselle, en quantité plus importante. Notons que ces considérations découlent d'observations de surface et pourraient être contredites par l'exploration stratigraphique des cavités, qui n'est pas, à l'heure actuelle, à l'ordre du jour.

\section{LE MOBILIER CÉRAMIQUE}

Plus de 800 tessons forment la collection de Punta di Casteddu. Tous issus de collectes superficielles, ils proviennent soit du secteur des habitations, soit du secteur des abris occidentaux. Dans ce dernier cas, le mobilier a été classé par cavité de provenance. L'ensemble du lot est composé de récipients montés au colombin à partir d'argiles probablement 
locales, dégraissées naturellement et/ou artificiellement avec des matériaux détritiques issus d'arènes granitiques. Les épaisseurs de paroi sont généralement assez stables sur un même vase. Les surfaçages sont assez soignés. Le brunissage est le traitement superficiel le plus fréquent. Les cuissons sont en grande majorité oxydantes, même si une minorité de vases, généralement ceux dont la matrice plastique est la plus épurée, présente un éclat noir, parfois rehaussé par polissage voire lustrage, correspondant à la «production B »du Bronze final méridional (Peche-Quilichini, 2014).

\section{LES FORMES DE LA VAISSELLE}

Plutôt que de présenter les lots contexte par contexte, nous avons privilégié une approche préliminaire par groupes typologiques afin de traiter la collection comme un ensemble, avant d'évoquer d'éventuelles spécificités, notamment du point de vue des associations. Les éléments dont la provenance n'est pas précisée ont été collectés dans le secteur des abris occidentaux, à l'extérieur des cavités.

\section{LES FORMES BASSES}

Fig. $4, n^{\circ} 1$ : assiette à paroi rectiligne ouverte achevée par une lèvre aplatie à convexe montée sur fond talonné assez épais. Diamètre à l'ouverture : environ $19 \mathrm{~cm}$.

Les assiettes à paroi rectiligne sont particulièrement nombreuses dans le sud de la Corse au cours du Bronze final et jusque dans les premiers temps de l'âge du Fer, soit un intervalle compris entre 1200 et 750 av. J.-C. (Peche-Quilichini, 2014). La présence d'un fond talonné ne semble pas avoir de valeur chronologique.

Fig. $4, n^{\circ} 2$ : abri 5 ; assiette/plat à paroi fine rectiligne et ouverte achevée par une lèvre aplatie, montée sur fond talonné obtenu par superposition de deux disques, d'où une épaisseur assez conséquente pour ce type de forme, contrastant avec celle de la paroi. Le disque inférieur est inséré dans un disque muni d'un pied en collerette et légèrement plus large. Ce dernier a été obtenu sur motte aplatie sur une vannerie discoïdale. Diamètre à l'ouverture : environ $24 \mathrm{~cm}$.

Comme la forme précédente, ce type de forme basse est récurrent durant le Bronze final et jusqu'au VIII ${ }^{\mathrm{e}}$ siècle (PecheQuilichini, 2014, cat. 3). L'association de la technique de superposition de deux disques de fond à celle de l'aplatissement sur vannerie est un unicum à l'échelle de l'île. L'utilisation de paillasses à tressage concentrique vise probablement la calibration du diamètre (Peche-Quilichini, 2009) et connaît des fréquences importantes jusqu'au Bronze final 3 mais quelques exemples sont connus jusqu'au $\mathrm{VI}^{\mathrm{e}}$ siècle sur certains sites (Nuciaresa ; PecheQuilichini et al., à paraître 1), notamment dans le cadre d'une fonctionnalité en tant que couvercle. En revanche, la technique de superposition de disques est plus caractéristique du Bronze final 3, comme dans le cas de la collection de l'abri 2 de Cuciurpula (Peche-Quilichini, 2014).

Fig. $4, n^{\circ} 3$ : fragment de fond aplati sur vannerie appartenant très probablement à une forme basse. Il s'agit de la partie périmétrale du fond, proche de l'accroche de la paroi. Son diamètre originel peut être estimé entre 22 et $28 \mathrm{~cm}$.

Comme énoncé plus haut, la technique employée pour réaliser ce fond renvoie plutôt au Bronze final (Peche-Quilichini, 2009).

\section{LES RÉCIPIENTS SUBCYLINDRIQUES VERTICAUX}

Fig. $4, \mathrm{n}^{\circ} 4$ : gobelet cylindrique à paroi légèrement convexe achevée par une lèvre aplatie à convexe. La hauteur du récipient ne devait pas dépasser 10 à $12 \mathrm{~cm}$. Diamètre à l'ouverture : $10 \mathrm{~cm}$.

Cette forme de structure simple est représentée à toutes les époques de la Protohistoire insulaire.

Fig. $4, n^{\circ} 5$ : récipient cylindrique à paroi épaisse et lèvre aplatie dont la hauteur peut être estimée à 20-30 $\mathrm{cm}$. Son corps porte une perforation cylindrique qui témoigne d'une réparation par suture. Diamètre à l'ouverture : environ $25 \mathrm{~cm}$.

Ici encore, la forme est trop simple pour fournir de bonnes occurrences chronologiques. On signale néanmoins quelques comparaisons efficaces dans le premier âge du Fer initial de Cuciurpula (Peche-Quilichini, 2014, cat. 27).

\section{LES RÉCIPIENTS OUVERTS HÉMISPHÉRIQUES OU TRONCONIQUES}

Fig. $4, n^{\circ} 6:$ abri 5 ; bol/écuelle tronconique à paroi rectiligne avec légère accentuation sub-labiale de l'évasement, à lèvre aplatie. Diamètre à l'ouverture : 14 à $16 \mathrm{~cm}$. La hauteur peut être estimée à 9-12 cm.

La forme est un standard des collections du Bronze final d'Apazzu Vecchju, de Torracone, de Ceccia et de Cucuruzzu (Peche-Quilichini, 2014, cat. 15). On la connaît aussi cependant au premier âge du Fer à Viccia (Peche-Quilichini, 2012a).

Fig. 4, no 7 : terrasse occidentale ; bol/jatte à paroi rectiligne très évasé achevée par une lèvre plate à épaississement interne. Diamètre à l'ouverture : environ $21 \mathrm{~cm}$. Hauteur estimée : 7 à $10 \mathrm{~cm}$.

Variante de la forme précédente, on trouve de bonnes comparaisons, toutefois dénuées de l'aménagement particulier de la lèvre, à Cucuruzzu, pour le Bronze final (Peche-Quilichini, 2014, cat. 15).

Fig. $4, n^{\circ} 8$ : vase à paroi sub-rectiligne légèrement évasée et achevée par une lèvre aplatie. Un cordon appliqué et vertical de section rectangulaire, très proéminent, court à quelques centimètres sous l'ouverture. Diamètre à l'ouverture estimé : $23 \mathrm{~cm}$. Une hauteur de 17 à $21 \mathrm{~cm}$ peut être envisagée.

Ce profil simple est un grand classique du Bronze final du sud de la Corse au sein des séquences les plus représentatives, telles Cucuruzzu, Punta Campana, Ceccia et Apazzu Vecchju (Peche-Quilichini, 2014, cat. 18). La présence d'un cordon est toutefois une originalité.

Fig. $4, n^{\circ} 9$ : abri 5 ; jatte à paroi légèrement convexe portant un rebord plus évasé, une lèvre convexe à inflexion externe et une angulation interne basse et proéminente aménagée en réceptacle. Diamètre à l'ouverture : environ $24 \mathrm{~cm}$. Hauteur estimée : 10 à $13 \mathrm{~cm}$.

$\mathrm{Si}$ le profil apparaît également comme particulièrement typique des contextes méridionaux du Bronze final, l'aménagement de la lèvre présente une certaine originalité que l'on ne retrouve, sous des formes légèrement divergentes, qu'à Cucuruzzu et, de manière plus évidente, à Ceccia (Peche-Quilichini, 2014, cat. 18). Une lèvre identique est en revanche connue pour le Bronze final, à Petra Pinzuta, mais pour une forme différente.

Fig. $4, \mathrm{n}^{\circ} 10$ : jatte à paroi convexe s'épaississant vers le bas et achevée par une lèvre aplatie à convexe. Le rebord est 

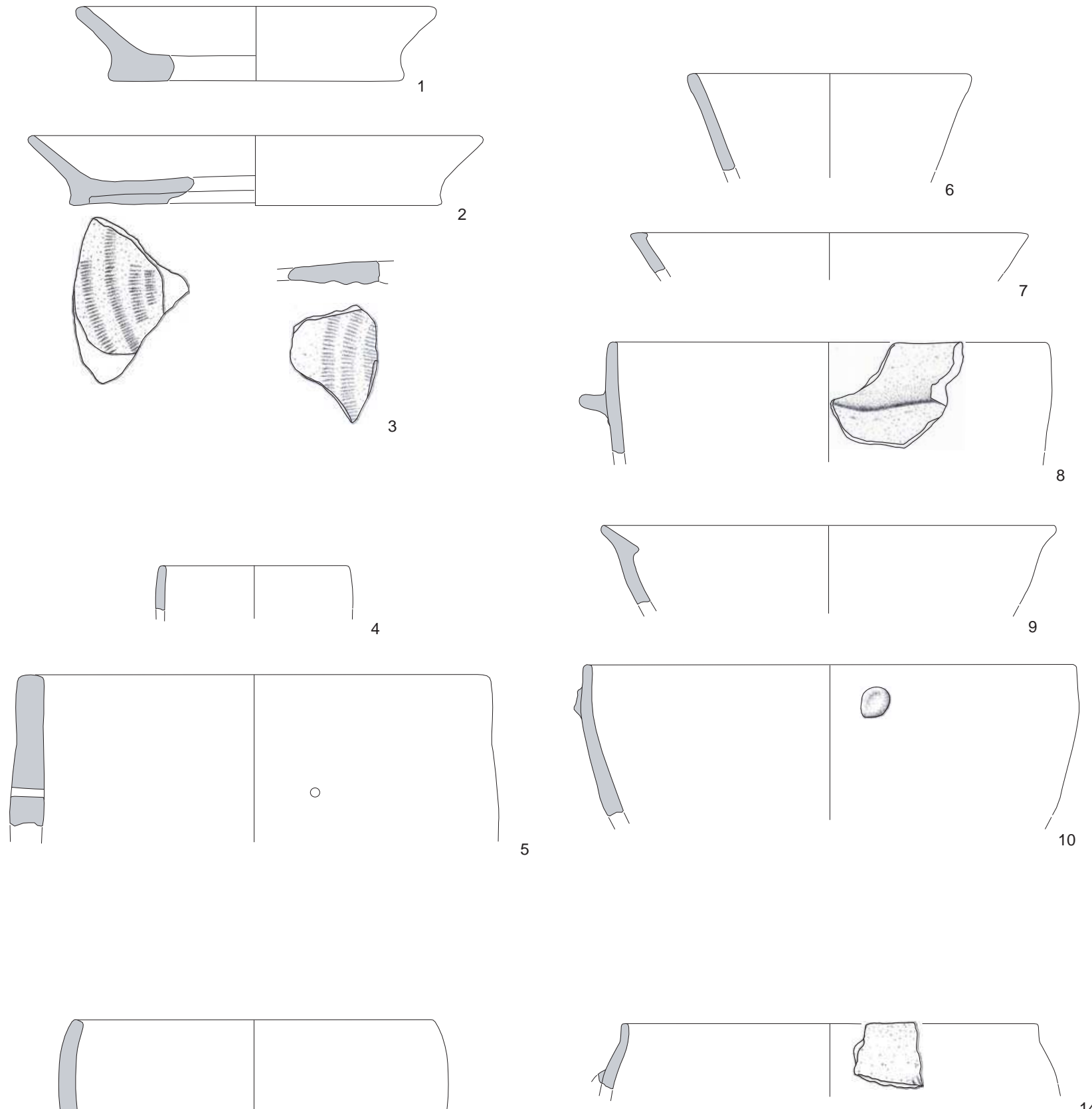

14

11
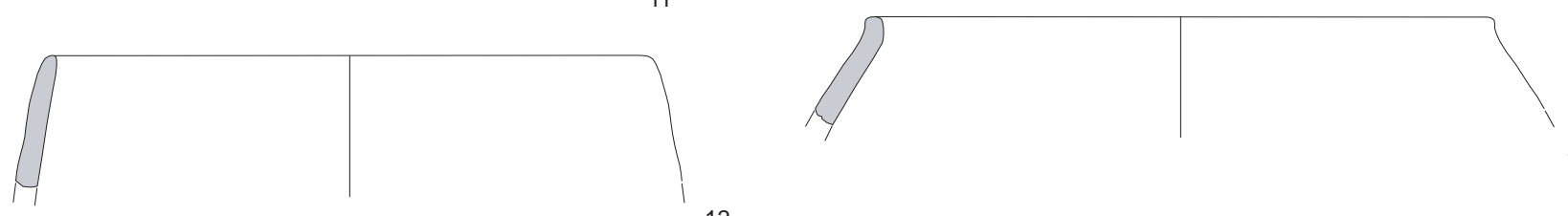

12

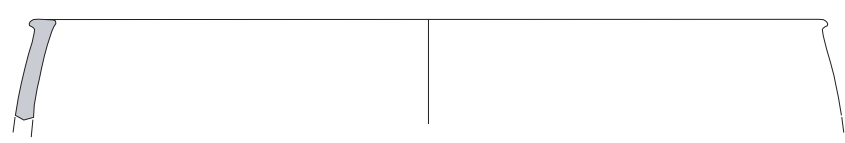

13

Fig. 4 - Mobilier céramique (DAO K. Peche-Quilichini, LAMPEA).

Ceramic assemblage (graphics: K. Peche-Quilichini, LAMPEA). 
cylindrique. À la base de ce dernier est appliquée au moins une pastille circulaire d'un diamètre de $2 \mathrm{~cm}$ dont le sommet est légèrement concave. Diamètre à l'ouverture : environ $26 \mathrm{~cm}$. Hauteur estimée : 11 à $14 \mathrm{~cm}$.

Il existe pour ce vase une bonne comparaison à Cucuruzzu, au Bronze final, où une languette se substitue à la pastille (Peche-Quilichini, 2014, cat. 16).

\section{LES RÉCIPIENTS OVOÏDAUX FERMÉS}

Fig. $4, n^{\circ} 11$ : vase hémisphérique aplati à lèvre plate à inflexion interne et partie supérieure à paroi convexe. Diamètre à l'ouverture : 18 à $20 \mathrm{~cm}$. Le récipient présentait une hauteur comprise entre 8 et $12 \mathrm{~cm}$.

Il existe de nombreuses analogies entre ce récipient et des exemplaires du Bronze final de Cucuruzzu ou Torracone et d'autres, datés du début du premier âge du Fer, de Cuciurpula (Peche-Quilichini, 2014, cat. 20).

Fig. $4, \mathrm{n}^{\circ} 12$ : vase ovoïdal à rebord rectiligne convergent achevé par une lèvre convexe. Le diamètre à l'ouverture peut être estimé autour de $22 \mathrm{~cm}$.

Ce rebord appartient vraisemblablement à un type de jarre diffusé entre l'étape moyenne du Bronze final et le milieu du premier âge du Fer, à Apazzu Vecchju, Furcina, Santa Barbara, Cucuruzzu et Cuciurpula (Peche-Quilichini, 2014, cat. 53).

Fig. $4, n^{\circ} 13$ : vase ovoïdal à rebord convergent légèrement convexe aboutissant à une lèvre aplatie à développement externe. Diamètre à l'ouverture : environ $28 \mathrm{~cm}$.

Variante de la forme précédente, l'aménagement particulier de la lèvre ne trouve d'équivalents qu'à Cucuruzzu, au Bronze final (Peche-Quilichini, 2014, cat. 53).

\section{LES RÉCIPIENTS OVOÏDAUX FERMÉS À PETIT COL VERTICAL}

Fig. $4, \mathrm{n}^{\circ} 14$ : secteur des habitations ; récipient ovoïdal à parie supérieure convergente à paroi convexe prolongée par un petit col vertical à lèvre aplatie. Collé au-dessus du diamètre maximal un dispositif de préhension arraché de forme indéterminée porte des incisions subverticales courtes. Le diamètre à l'ouverture de ce vase est estimé à $22 \mathrm{~cm}$.

Ce type de récipient connaît une grande diffusion sur tous les sites du Bronze final 3 et du premier âge du Fer dans le sud de la Corse, notamment à Cucuruzzu, à Viccia et à Cuciurpula (Peche-Quilichini, 2014, cat. 48). La présence d'un élément appliqué portant des incisions plaide de façon assez décisive pour une attribution chronologique au premier âge du Fer.

Fig. $4, n^{\circ} 15$ : récipient ovoïdal à partie supérieure très légèrement convexe et prolongée par un petit col vertical à lèvre aplatie. Diamètre à l'ouverture : environ $23 \mathrm{~cm}$.

Ce type de profil se développe dans le sud de la Corse à la fin du Bronze final et tout au long du premier âge du Fer (Peche-Quilichini, 2014, cat. 48).

\section{Les réCIPIENTS ARTICUlés et FERMÉS À PETIT COl ÉVASÉ}

Fig. $5, \mathrm{n}^{\circ} 1$ : abri 1 ; récipient à profil bitronconique probablement non segmenté dont la partie supérieure est concave et donne naissance à un col évasé non articulé et achevé par une lèvre aplatie à forte inflexion externe. Cette lèvre porte une à deux excroissance(s) appliquée(s) en forme de languette pointant légèrement au-dessus de l'horizontale. Diamètre à l'ouverture estimé : $16 \mathrm{~cm}$. On suppose une partie inférieure hémisphérique à fond plat, pour une hauteur minimale de $20 \mathrm{~cm}$.

Il s'agit d'un profil caractéristique des étapes médiane et terminale du Bronze final méridional, qui perdure toutefois jusque dans un temps avancé du premier âge du Fer (PecheQuilichini, 2014, cat. 62). Les meilleures comparaisons sont fournies par les séquences de Cucuruzzu, de Torre, de Torracone, de Ceccia et de la Punta di a Villa.

Fig. $5, \mathrm{n}^{\circ} 2$ : récipient en tonneau à petit col évasé et lèvre aplatie dont le diamètre à l'ouverture est proche de $20 \mathrm{~cm}$. À $4 \mathrm{~cm}$ sous l'ouverture est appliqué un bouton hémisphérique.

Il existe des formes approchantes dans le premier âge du Fer initial de Cuciurpula (Peche-Quilichini, 2014, cat. 62).

Fig. $5, n^{\circ} 3$ : abri 7 ; vase ovoïdal à paroi supérieure légèrement convexe et col légèrement évasé terminé par une lèvre aplatie. Diamètre à l'ouverture : environ $20 \mathrm{~cm}$.

Comme le vase précédent, les meilleures comparaisons disponibles pour ce profil proviennent de Cuciurpula, pour le premier âge du Fer. Un autre récipient morphologiquement proche est connu sur le site d'A Cota (Peche-Quilichini, 2014, cat. 62). Un exemplaire d'Alo-Bisughjè, dont le profil est voisin, provient cependant des niveaux du Bronze final de ce site.

Fig. 5, $\mathrm{n}^{\circ} 4$ : abri 7 ; récipient d'une grande finesse, de profil probablement ovoïdal, à partie supérieure convergente subrectiligne et petit col droit, articulé et évasé, terminé par une lèvre aplatie à inflexion externe. Le diamètre à l'ouverture de ce vase, pour lequel on note un effort de symétrie, peut être estimé à $22 \mathrm{~cm}$.

Des profils strictement superposables existent à Cucuruzzu au Bronze final (Peche-Quilichini, 2014, cat. 62). Des comparaisons moins évidentes caractérisent des exemples plus anciens, provenant d'Apazzu Vecchju.

Fig. 5, n 5 : abri 9 ; jarre dont le corps est probablement ovoïdal ou bitronconique, à col articulé et évasé, achevé par une lèvre aplatie à inflexion externe. Le diamètre à l'ouverture est compris entre 38 et $42 \mathrm{~cm}$.

Il s'agit là du profil-type du rebord des jarres du Bronze final dans tous les contextes de Corse méridionale (PecheQuilichini, 2014, cat. 61).

\section{AUtres FORMES}

Fig. $5, \mathrm{n}^{\circ} 6$ : abri 5 ; fragment mésial d'un récipient à profil en $\mathrm{S}$ dont le diamètre maximal à la panse est d'environ $33 \mathrm{~cm}$.

Sans la partie supérieure, il est difficile de relier cet élément à un profil typologique précis. Néanmoins, la forme trouve des analogies dans plusieurs types de gros récipients du Bronze final et du premier âge du Fer de Corse du sud, comme certains provenant de Santa Barbara (Peche-Quilichini, 2013a, fig. 2 $\left.\mathrm{n}^{\circ} 1\right)$.

Fig. 5 - Mobilier céramique (DAO K. Peche-Quilichini, LAMPEA).

Ceramic assemblage (graphics: K. Peche-Quilichini, LAMPEA). 
Note préliminaire sur le site protohistorique de Punta di Casteddu (Sartène, Corse-du-Sud)
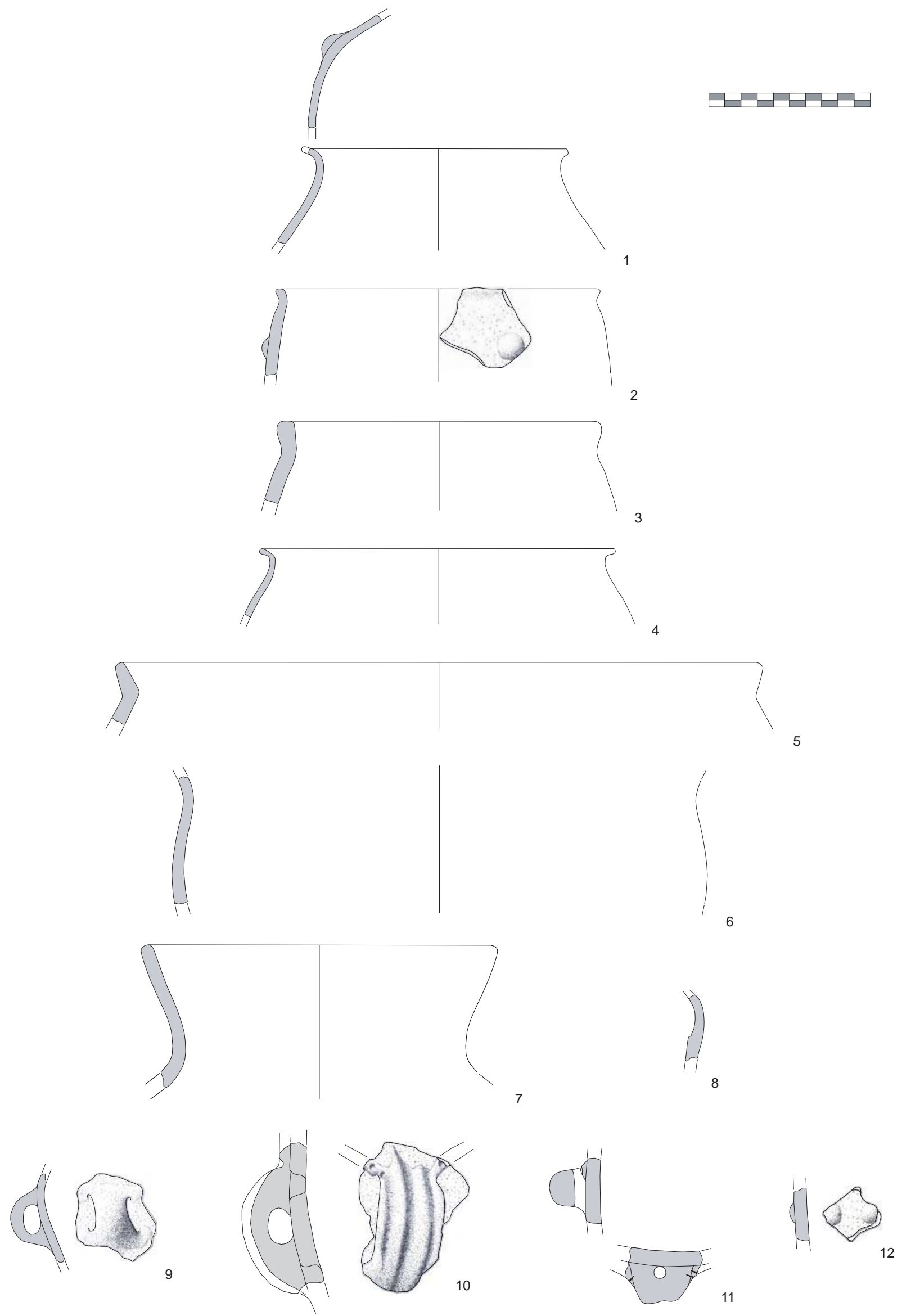

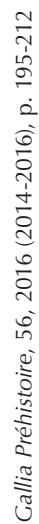

205 
Fig. $5, \mathrm{n}^{\circ} 7$ : abri 4 ; partie supérieure d'une bouteille à col large et divergent dont le diamètre à l'ouverture, déterminée par une lèvre aplatie, est d'environ $22 \mathrm{~cm}$. Le diamètre de l'étranglement, qui définit un corps probablement ovoïdal, est estimé à $18 \mathrm{~cm}$

Il s'agit d'une forme marginale des contextes du milieu ou de la fin du Bronze final méridional. On la connaît notamment à Cuciurpula, secteur nord-occidental, terrasse 4 (PecheQuilichini et al., 2014b). Le profil trouve un parallèle à la même époque en Sardaigne septentrionale, notamment dans les collections du nuraghe de la Prisgiona (Antona, 2012, fig. 3, $\mathrm{n}^{\circ}$ 4-5).

Fig. 5, n 8 : fragment de partie supérieure de récipient probablement piriforme dont la carène est surlignée par une cannelure superficielle. La zone orificielle est manquante pour environ deux centimètres de développement vertical. Un diamètre maximal d'environ 25 à $30 \mathrm{~cm}$ peut être envisagé pour le changement d'inflexion.

Ce type de carène cannelée sur structure générale piriforme est connu à Torre, Torracone, Cucuruzzu et Capula au Bronze final (De Lanfranchi, 1978, fig. 38, n 4 ; Peche-Quilichini, 2014, cat. 60). Il en existe aussi des exemples dans le Bronze moyen (ou final ?) de Tusiu (De Lanfranchi, 1998, fig. 5, $\left.n^{\circ} 4-5\right)$.

\section{LES DISPOSITIFS DE PRÉHENSION DÉTACHÉS DU CORPS DU RÉCIPIENT}

Fig. 5, $\mathrm{n}^{\circ} 9$ : anse rubanée de section subrectangulaire pour laquelle il n'a pas été possible de déterminer la présence d'un ou plusieurs tenon(s) d'ancrage.

Les anses rubanées sont présentes tout au long de la Protohistoire corse, avec des fréquences toutefois largement plus hautes au cours de l'âge du Bronze.

Fig. $5, n^{\circ} 10$ : anse rubanée maintenue sur la panse par deux tenons d'ancrage placés dans le prolongement du ruban. La partie externe du dispositif de préhension porte quatre cordons verticaux étendus jusqu'à la zone de collage de l'anse sur la paroi. La partie supérieure du ruban est prolongée par deux cordons, probablement arciformes, portant des impressions circulaires non réalisées au doigt.

$\mathrm{La}$ forme du ruban est particulièrement originale et ne trouve aucun équivalent dans l'île. Néanmoins, la présence des quatre cordons, qui donnent naissance à trois sillons creux, rappellent la mode de l'ensellement longitudinal des dispositifs de préhension qui caractérise le sud de l'île au premier âge du Fer. La présence des deux cordons impressionnés, éléments totalement absents des corpus du Bronze final, évoque les prises cornues qui apparaissent en contexte particulier (sépultures et dépôts de fondation) à partir du viII ${ }^{\mathrm{e}}$ siècle dans les vallées méridionales (Peche-Quilichini, 2010a, 2014, cat. Id ; RecchiaQuiniou et al., à paraître).

Fig. 5, $\mathrm{n}^{\circ} 11$ : secteur des habitations ; anse massive ${ }^{4}$ à perforation cylindrique verticale à laquelle répond, sur la partie externe, un léger ensellement vertical et parallèle. Il est

4. Nous utilisons ce terme pour caractériser des dispositifs de préhension faisant intervenir une perforation réalisée «dans la masse » par introduction d'un outil et non pas par pliage comme dans le cas des anses rubanées. impossible de savoir si cet ajout, de forme semi-rhomboïdale, est maintenu par un système de tenon/mortaise. En partie haute, l'anse est prolongée par un cordon vertical incisé venu s'appuyer dessus. Il est fort probable qu'une autre prise du même type devait être présente, en opposition diamétrale, sur le récipient.

De nombreuses prises identiques sont connues à Cuciurpula, à Magazene et à Viccia au premier âge du Fer (Peche-Quilichini, 2014, cat. Ib).

Fig. 5, $\mathrm{n}^{\circ} 12$ : abri 10 ; fragment de panse portant deux boutons hémisphériques et appartenant vraisemblablement à une ligne horizontale ceinturant le récipient.

L'alignement horizontal de boutons hémisphériques se retrouve dans la collection de la sépulture voisine d'Acciola, pour un horizon chronologique à situer vers les VIII $/ \mathrm{VII}^{\mathrm{e}}$ siècles (Peche-Quilichini, 2014, cat. V).

\section{CARACTÉRISATION CHRONOLOGIQUE D'ENSEMBLE}

À l'exclusion de quelques éléments ubiquistes, la série céramique de Punta di Casteddu inclut exclusivement du mobilier daté par cross-dating entre le milieu du Bronze final et le milieu du premier âge du Fer. Malgré sa constitution par ramassages superficiels et zonés, elle apparaît donc comme homogène et peut être replacée dans l'intervalle 1100-700 av. J.-C. L'absence d'occurrence pour le Bronze récent et le Bronze final 1, ainsi que celle du décor « en grains de riz », typique des ambiances méridionales à partir de la fin $\mathrm{du} \mathrm{VIII}^{\mathrm{e}}$ siècle, valide cette hypothèse chronologique. À la condition que l'échantillonnage de surface soit représentatif des durées d'occupation, la spatialisation des données tendrait plutôt à accorder aux abris du secteur nord-ouest du massif un fonctionnement plus précoce que celui du secteur des habitations, avec une distribution de vestiges attribuables au premier âge du Fer autour de ces dernières alors que les tessons qui semblent plus caractéristiques de la fin du $\mathrm{II}^{\mathrm{e}}$ millénaire proviennent des abris. Deux occupations a priori fonctionnellement différenciées seraient donc également chronologiquement différées, bien que partiellement contemporaines.

\section{HYPOTHÈSES FONCTIONNELLES ET THÉORIE SUR L'INCINÉRATION}

Si l'interprétation des deux structures ovalaires formées de blocs de granit en tant qu'habitations ne peut plus aujourd'hui être sujette à caution (Milletti et al., 2012 ; Peche-Quilichini et al., 2012, 2013, 2014b), la question de l'utilisation des abris reste ouverte. On note ici que leur typologie et leur superficie sont extrêmement variées. Certains, vastes (jusqu'à près de $30 \mathrm{~m}^{2}$ ), sont bas de plafond ; d'autres sont réduits à d'étroits corridors peu praticables ; plusieurs présentent un espace appréciable. Tous livrent de grandes quantités de mobilier céramique. Les récipients de stockage sont absents, ce qui est assez étonnant en regard de données récentes obtenues en d'autres cavités de l'île, utilisées de façon contemporaine précisément pour y placer les grands contenants (site de Tuani, commune de Corté, fouilles 
N. Federzoni). À Punta di Casteddu, hormis quelques récipients de gabarit moyen (fig. 5, $n^{\circ} 5-6$ ), la vaisselle inclut uniquement des petits vases, soit un set de table et de service. Pour certaines cavités, particulièrement riches en mobilier $^{5}$, pouvant être qualifiées d'anfractuosités plutôt que d'abris-sous-roche ou de grottes naturelles, la fonction habitative et/ou liée à un caractère fonctionnel d'un habitat voisin est souvent à exclure en raison de leur exiguïté. Dès lors, la possibilité d'une considération générale de cette partie du site comme un regroupement de sépultures peut être envisagée.

Il faut rappeler ici que la tradition de l'utilisation des taffoni et des sapari (toponymie liée aux cavités granitiques creusées par l'érosion et typiques des paysages du sud de la Corse et du nord de la Sardaigne) remonte au Mésolithique (Сатри Stefanu ; Courtaud et al., 2014), caractérise tout le Néolithique/Bronze ancien (entre autres exemples : Torre d'Aquila, I Calanchi-Sapar'Alta, San Michele ; Cesari, 1986 ; David, 2000 ; Magdeleine, 1995) et perdure tout au long de l'âge du Fer, comme à Tappa 2, Lugo, Santa Catalina ou Grotta Piatta (De Lanfranchi, 1974 ; Marchetti, 2007 ; Milanini et al., 2008), voire après, et semble coexister avec l'utilisation de caveaux mégalithiques dès le Néolithique moyen, au Monte Revincu (Leandri et al., 2012), et jusqu'au début de l'âge du Fer, à Mamucci (Gilabert, Leandri, 2004). Sur ce point particulier, on rappellera qu'en Sardaigne septentrionale, on a pu démontrer l'utilisation simultanée de «tombes de géants » et de taffoni durant la seconde moitié de l'âge du Bronze, marquée par une nette discrimination dans le recrutement entre les deux types de sépultures : individus jeunes, rachitiques et témoignant d'importantes carences dans les grottes naturelles, population à distribution d'âge « normale » et ne laissant voir aucune tendance sanitaire particulière dans les tombes mégalithiques (Germanà, 1999). Dans cette région, à cette époque, il existerait donc une distinction (d'ordre social ?) entre deux groupes cultivant des considérations sépulcrales divergentes, à moins que le choix de la dernière demeure n'ait été défini que post mortem, en fonction de la raison du décès (Depalmas, 2009). En Corse, si l'on est loin d'arriver à de telles conclusions, c'est parce qu'aucun contexte sépulcral du Bronze moyen et final n'a été individualisé à ce jour. En effet, aucune sépulture n'est connue entre le Bronze ancien 2 (tombes sous abri de Minza et de Murteddu ; Grosjean et al., 1976) et le milieu du premier âge du Fer (abri 2 de Cuciurpula, abri 1 d'Acciola, voire grottes de Magazene et de Viccia ; Milletti et al., 2012 ; Peche-Quilichini, 2012a, 2012c), à l'exception d'une utilisation du coffre semi-mégalithique de Cuntrasarda, datée du XIII ${ }^{\mathrm{e}}$ siècle par une datation radiocarbone et qui a livré quelques tessons attribuables au Bronze récent (Peche-Quilichini, 2014), mais dont on ne sait rien des individus associés.

Dans ce contexte historiographique lacunaire, l'adoption massive, de la part des groupes du sud de la Corse, du prototype nord-italique du vase biconique à court col évasé, apporte peut-être un élément de réponse. En Piémont, Ligurie

5. Les abris ont aussi livré des fragments brûlés d'os de porc et de bœuf, identifiés par Elena Bedini (Anthropozoologica - Laboratorio di Antropologia e Bioarcheologia S.N.C.). et Toscane ${ }^{6}$, à partir du Bronze récent 2 et surtout du Bronze final $1^{7}$, se diffuse le rite de l'incinération, suivie d'une collecte des cendres dans une urne biconique à rebord ouvert, du même type que les récipients contemporains découverts sur tous les sites corses du Bronze final. En ce sens, si les infiltrations culturelles nord-italiques ont dépassé le simple cadre de l'imitation matérielle et ont touché des sphères ancrées plus profondément dans les considérations socio-cultu(r)elles des communautés du sud de la Corse, rien n'interdit de penser que l'introduction, même partielle, du rite crématoire ait pu toucher cette partie de l'île vers 1200 av. J.-C., au moment de la diffusion de ces pratiques dans l'espace nord-tyrrhénien ${ }^{8}$. Punta di Casteddu pourrait donc illustrer une forme de syncrétisme entre un rite extérieur et la tradition millénaire de placer les défunts dans les grottes naturelles. À ce titre, la reconnaissance d'une urne (Peche-Quilichini, 2014, fig. 461) provenant du site voisin de Punta Campana, qui présente un décor proche de ceux développés sur les contenants cinéraires des nécropoles de campi d'urni proto-Golasecca du Piémont, est particulièrement éloquente, même si le contexte de découverte reste obscur. Ces conclusions demeureront hypothétiques tant que des fouilles précises ne seront pas menées sur des gisements susceptibles d'appartenir à ce courant ${ }^{9}$.

\section{TERRITOIRES ET IDENTITÉ DANS LE SUD DE LA CORSE DURANT LA TRANSITION BRONZE/FER}

Punta di Casteddu est un site archéologique utilisé durant la transition Bronze/Fer, soit un moment où la densité démographique, déduite d'après le nombre conséquent de sites sartenais occupés durant cet intervalle chronologique, connaît un développement sans précédent, en termes de temps comme d'espace

6. Un cas a récemment été individualisé en Provence pour le Bronze récent (soit le Bronze final I de la chronologie traditionnelle du sud de la France ; Aujaleu et al., 2013). Pour l'Italie, les exemples sont particulièrement nombreux ; entre autres : Bietti Sestieri et al., 2001 ; D’Ambrosio, 1987, fig. 12, n 7 ; Del Lucchese, Maggi, 1985, fig. 8, $\mathrm{n}^{\circ} 1$; Di Gennaro, 1986, p. 79 ; Domanico, 1998, fig. 2 ; Negroni Catacchio, 1998, fig. 1 ; Pacciarelli, 2001 ; Rittatore Vonwiller, 1975 ; Rubat Borel, 2006 ; Venturino Gambari, 2006, fig. 12.

7. L'adoption de l'incinération se fait plus précocement en ÉmilieRomagne, au moins dès le Bronze moyen 2 chez les groupes terramaricoles (Cardarelli, Tirabassi, 1997).

8. À Punta di Casteddu, certains récipients (fig. 5, $\mathrm{n}^{\circ} 1,4,5$ ) rappellent, à différents degrés, les urnes nord-italiques. Dans les contextes padans et toscans, ces récipients sont systématiquement fermés par une écuelle retournée. En Corse, la fermeture a pu se faire à l'aide d'une assiette ou d'un plat, dont on connaît par ailleurs l'ambivalence fonctionnelle (Peche-Quilichini, 2009, à paraître).

9. À noter que le site de Mamucci (Santo-Pietro-di-Tenda, HauteCorse), fouillé au début des années 2000 (Gilabert et Leandri, 2004), inclut deux tumulus à ciste central ayant probablement accueilli des restes humains calcinés dans des urnes à cordon. Un cercle de pierre (bustum ?) a également été fouillé. Cet ensemble remarquable, associé à un groupe de statues-menhirs, est datable à une époque indéterminée du Bronze final et/ou du premier âge du Fer. 
(Peche-Quilichini, 2012a, 2012b, 2015) (fig. 6). Les habitats occupés en $900 \mathrm{BC} \pm 200$ sont particulièrement nombreux en proximité immédiate : U Grecu, Punta Campana, Santa Barbara, Torracone, Valchiria, pour ne citer que ceux occupés au Bronze final et au premier âge du Fer et en situation de covisibilité. Punta di Casteddu, comme ces derniers, présente une morphologie de sommet où les affleurements massifs sont rythmés par des replats où s'accumulent les dépôts détritiques et bénéficiant d'une vigie sur les plaines alluviales et les plateaux voisins. L'articulation inter-sites est dictée par la géologie de cette partie du Sartenais, où les vallées sont toujours sensiblement parallèles à celle de l'Ortolu, avec des cours d'eau se déversant du nord/nord-est vers le sud/sud-ouest. La récurrence du système d'implantation à cette époque dans cette partie de l'île est donc un fait avéré et doit être considérée comme une partie d'un système au sein duquel les alignements de pierres dressées et autres monolithes isolés jouent également un rôle dans la perception et l'utilisation du territoire (D'Anna et al., 2006a ; Soula, 2012a, 2012b). À ce titre, la reconnaissance récente de villages incluant un modèle systématique d'organisation nucléaire de petites habitations aux soubassements massifs constitués de blocs de granit n'est pas anodine. Elle se superpose pleinement aux acquis dernièrement développés dans la microrégion située immédiatement en amont des cours de l'Ortolu et du Rizzanese, l'Alta Rocca (Peche-Quilichini et al., 2015). Dans ces hautes vallées, la reconnaissance d'entités telles Cuciurpula, Puzzonu, Nuciaresa ou Cumpulaghja trouve en aval son écho dans les identifications réalisées ces dernières années autour de la vallée de l'Ortolu, à Briccu, Funtanedda,
Punta Campana, Canava, Valchiria, Middari, Baricci et donc Punta di Casteddu. Bien que les écosystèmes soient différents, le schéma d'occupation des sols semble au moins en partie superposable et il paraît déjà envisageable de l'étendre à des secteurs tels le bassin de Porto-Vecchio/Pian d'Avretu. Cette relative unité d'ordre socio-économique répond à l'homogénéité matérielle constatée pour ces régions. Les témoignages offerts par le mobilier céramique, seule sphère productive qualitativement quantifiable et quantifiée sur l'ensemble des territoires concernés, rendent comptent, hors spécificités fonctionnelles, de la remarquable uniformité culturelle qui unit les groupes de Corse méridionale à la charnière entre âges du Bronze et du Fer. Dans les faits, l'ensemble des séquences mobilisées permet d'observer le passage graduel entre les industries de type Apazzu-Castidetta-Cucuruzzu du Bronze final et celles de type Nuciaresa du premier âge du Fer (Peche-Quilichini, 2010b, 2012a, 2012c, 2013a). Tous les signaux convergent donc vers une considération unitaire des groupes de la Protohistoire moyenne des vallées du sud de l'île, et ce, malgré des emprunts ponctuels aux régions voisines, Sardaigne, nord de la Corse ou côtes tyrrhéniennes de l'Italie. Plus au nord, à peine franchi le Taravu, véritable marge culturelle à partir de la fin du $\mathrm{II}^{\mathrm{e}}$ millénaire, productions architecturales et matérielles révèlent des divergences toujours plus affirmées selon un gradient sud-nord évident. Ainsi, au rythme d'investigations archéologiques dont les contours administratifs et les problématiques d'étude sont différents mais toujours a posteriori complémentaires, le sud de la Corse affirme son originalité et permet d'approcher toujours un peu plus la notion d'identité.

Fig. 6 - (DAO K. Peche-Quilichini, LAMPEA). Sites domestiques et sépulcraux : 1 - Punta di Casteddu ; 2 - U Grecu ; 3 - Middari ; 4 - Petra Nera ; 5 - Ranfonu-Giovichi ; 6-Punta di Casteddu 2; 7 - Bariccini ; 8 - Punta Campana ; 9 - Furcina ; 10 - Castidetta-Pozzone ; 11 - Santa Barbara ; 12 - Minza ; 13 - Baresi ; 14 -Viddafranca ; 15 - Zivoli ; 16-Aravu ; 17 - Alo-Bisughjè ; 18 - Calanchedda ; 19 - Casteddu di Grossa ; 20 - Vinturossu ; 21 - Gianfutru ; 22 - A Coscia ; 23 - Valchiria ; 24 - Canava ; 25 - Castidducciu di Vaccil’Vecchju ; 26 - Quarcioqua ; 27 - Bavoghja ; 28 - Acciola ; 29 - A Cota ; 30 - A Viccia ; 31 - Magazene ; 32 - Apazzu ; 33 - Petra Pinzuta ; 34 - Patania ; 35 - A Villa ; 36 - Turrione ; 37 - Castiddacciu ; 38 - Tepparedda; 39 - Sapara d'Acquedda ; 40 - plage d'Acquedda ; 41 - Ranfone; 42 - Plateau de Cauria, divers indices d'occupation. Sites à pierres dressées : a. Alignement de Bocca di a Pila intégrant au moins un menhir-stèle armé; $\boldsymbol{b}$. Alignement de Palaghju intégrant des menhirs-stèles armés ; c. Alignement d'Apazzu intégrant des statues-menhirs ; d. Alignement d'I Stantari intégrant des statues-menhirs et des menhirs-stèles armés; e. Alignement de Rinaghju intégrant un menhir-stèle armé ; f. Statue-menhir et menhirs de Petra Pinzuta, vestiges d'un probable alignement; g. Alignement de Curghja intégrant une statue-menhir, disparu anciennement ; $\boldsymbol{h}$. Statuemenhir de Castidetta-Pozzone ; i. Alignement de Muntagnola intégrant une statue-menhir ; j. Statue-menhir de Rizzanese-Polmona, disparue anciennement ; . Alignement de pierres dressées de Vinturossu ; l. Menhir isolé de Valchiria ; m. Menhir d'Alo-Bisughjè ; n. Menhir isolé disparu de Viddafranca ; o. Groupe de menhirs anciennement signalé à Santa Barbara.

(graphics: K. Peche-Quilichini, LAMPEA). Domestic and funerary sites: 1 - Punta di Casteddu; 2 - U Grecu; 3 - Middari; 4 - Petra Nera; 5 - Ranfonu-Giovichi; 6 - Punta di Casteddu-2; 7 - Bariccini; 8 - Punta Campana; 9 - Furcina; 10 - Castidetta-Pozzone; 11 - Santa Barbara; 12 - Minza; 13 - Baresi; 14 - Viddafranca; 15 - Zivoli; 16 - Aravu; 17 - Alo-Bisughjè; 18 - Calanchedda; 19 - Casteddu di Grossa; 20 - Vinturossu; 21 - Gianfutru; 22 - A Coscia; 23 - Valchiria; 24 - Canava; 25 - Castidducciu di Vaccil'Vecchju; 26 - Quarcioqua; 27 - Bavoghja; 28 - Acciola; 29 - A Cota; 30 - A Viccia; 31 - Magazene; 32 - Apazzu; 33 - Petra Pinzuta; 34 - Patania; 35 - A Villa; 36 - Turrione; 37 - Castiddacciu; 38 - Tepparedda; 39 - Sapara d'Acquedda; 40 - Acquedda beach; 41 - Ranfone; 42 - Cauria plateau, various traces of occupation. Sites with upright stones: a. Bocca di a Pila stone alignment with at least one menhir-stela featuring weaponry; $b$. Palaghju stone alignment with menhirsstelae featuring weaponry; c. Apazzu stone alignment with statues-menhirs; $d$. I Stantari stone alignment with statues-menhirs and menhirsstelae featuring weaponry; e. Rinaghju stone alignment with one menhir-stela featuring weaponry; $f$. Petra Pinzuta statue-menhir and menhirs, probably the remains of a stone alignment; g. Curghja stone alignment with one statue-menhir, traces lost; $h$. Castidetta-Pozzone statue-menhir; $i$. Muntagnola stone alignment with one statue-menhir; j. Rizzanese-Polmona statue-menhir, now lost; $k$. Vinturossu stone alignment; I. Valchiria, isolated menhir; m. Alo-Bisughjè menhir; $n$. Viddafranca, isolated menhir, now lost; o. Group of menhirs previously recorded at Santa Barbara. 
Note préliminaire sur le site protohistorique de Punta di Casteddu (Sartène, Corse-du-Sud)

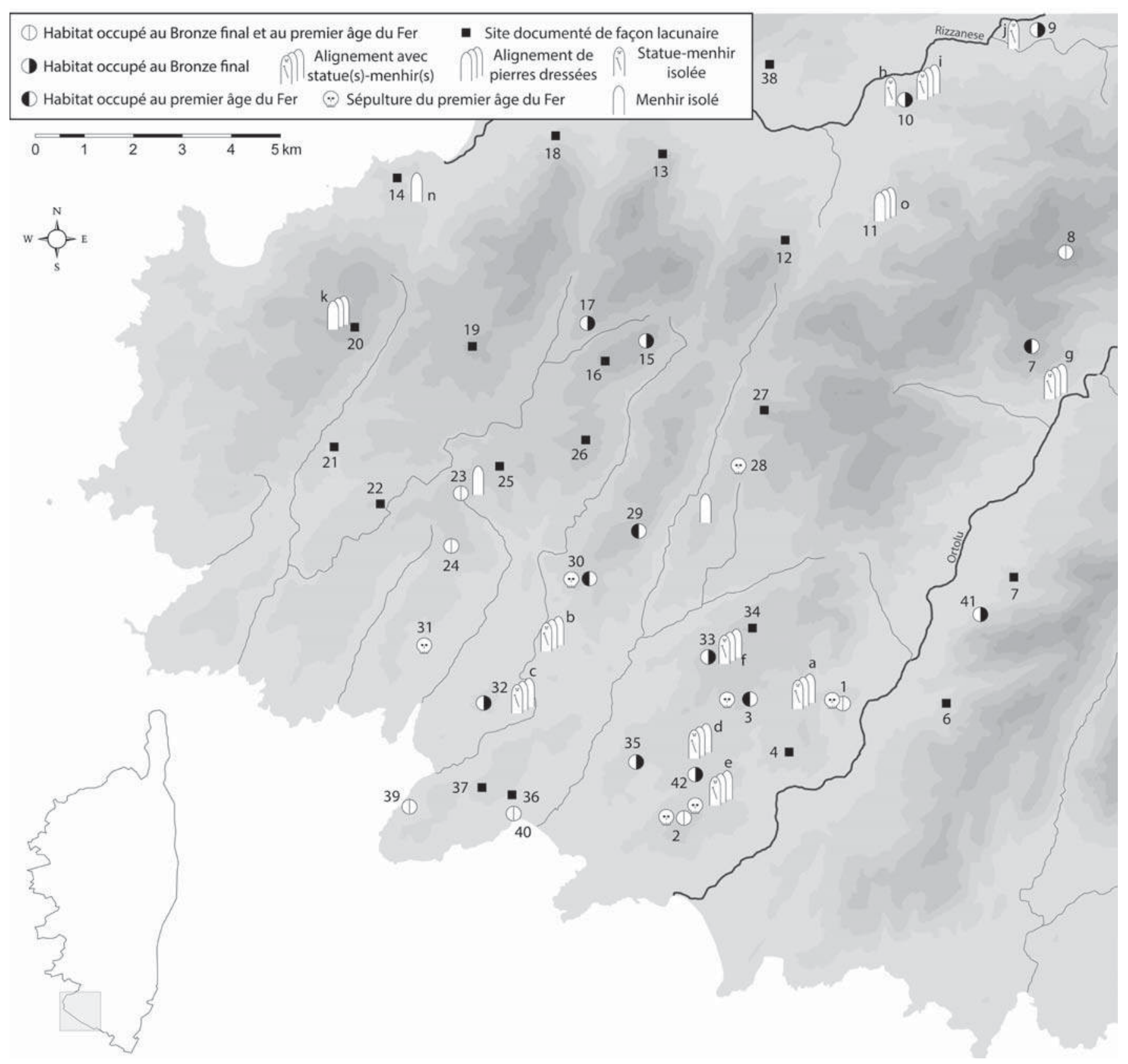




\section{BIBLIOGRAPHIE}

Antona A.

2012: Nota preliminare sui contesti stratigrafici della Gallura nuragica. L'esempio di La Prisgiona di Arzachena, dans : Preistoria e Protostoria della Sardegna, Atti della XLIV Riunione Scientifica dell'Istituto Italiano di Preistoria e Protostoria (Cagliari, novembre 2009), Florence, p. 687-696.

Aujaleu A., Granier G., Lachenal T.

2013: Un ensemble funéraire du début du Bronze final à Aix-en-Provence (Bouches-du-Rhône) : les sépultures secondaires à crémation du site du Conservatoire, Bulletin de la Société Préhistorique Française, CX, p. 719-743.

Bietti Sestieri A.M., De angelis M.C., Negroni Catacchio N., Zanini A.

2001: La Protostoria della Toscana dall'età del Bronzo recente al passaggio alla prima età del Ferro, dans : Preistoria e Protostoria della Toscana, Atti della XXXIV Riunione Scientifica dell'Istituto Italiano di Preistoria e Protostoria (Florence, septembre-octobre 1999), Florence, p. 91-115.

Cardarelli A., Tirabassi J.

1997: Le necropole delle terramare emiliane, dans : Bernabò-Brea M., Cardarelli A., Cremaschi M. (Dir.), Le terramare. La più antica civiltò padana, Electa, Milan, p. 677-697.

Cesari J.

1986: Le Néolithique et le Chalcolithique du gisement des Calanchi, Bulletin de la Société des Sciences Historiques et Naturelles de la Corse, 652, p. 319-358.

Courtaud P., Cesari J., Leandri F., Nebbia P., Perrin T., Petersen H.C., Zemmour A.

2014: La sépulture mésolithique de Campu Stefanu (Sollacaro, Corse-du-Sud, France), dans : Perrin T., SÉnépart I., Leandri F., Cauliez J. (Dir.), Chronologie de la Préhistoire Récente dans le Sud de la France. Acquis 19922012. Actualités de la recherche. Actes des $X^{e}$ Rencontres Méridionales de Préhistoire Récente (Ajaccio, octobre 2012), AEP, Toulouse, p. 275-287.

Codaccioni L.

2000: Atlas préhistorique de la commune de Sartène, Mémoire de maîtrise, Université de Provence, Aix-enProvence, $464 \mathrm{p}$.
D'Ambrosio B.

1987: Lo strato F della necropoli di Chiavari. Testimonianze di un sito costiero dell'età del Bronzo finale, Rivista di Studi Liguri, LIII, p. 5-76.

D’Anna A., Guendon J.-L., Pinet L., Tramoni P.

2006a: Espaces, territoires et mégalithes : le plateau de Cauria (Sartène, Corsedu-Sud) au Néolithique et à l'âge du Bronze, dans : Impacts interculturels au Néolithique moyen, $25^{\mathrm{C}}$ supplément à la R.A.E, S.A.E, p. 191-213.

D'Anna A., Guendon J.-L., Orsini J.-B., Pinet L., Tramoni P.

2006b: Nouvelles recherches sur les alignements mégalithiques d'I Stantari (Sartène, Corse-du-Sud), dans : Actes des VI ${ }^{e}$ Rencontres Méridionales de Préhistoire Récente (Périgueux, novembre 2004), Coédition ADRAHPPSO, p. 455-472

2007a: Les alignements mégalithiques du plateau de Cauria (Sartène, Corse-duSud), dans : D'Anna A., Cesari J., Ogel L., Vaquer J. (Dir.), Corse et Sardaigne préhistoriques : relations et échanges dans le contexte méditerranéen. Actes du $128^{\circ}$ Congrès du CTHS (Bastia, avril 2003), CTHS, Paris, p. 211-223.

2007b : Le plateau de Cauria du Néolithique à l'âge du Bronze, de la lecture événementielle à l'approche pluridisciplinaire anthropologique, hommage à Roger Grosjean, dans : Evin J. (DIR.), Congrès du centenaire : un siècle de construction $d u$ discours scientifique en Préhistoire. Vol. 1: Des idées d'hier... (Avignon, septembre 2004), volume II, SPF, Paris, p. 331-346.

DAVID H.

2000 : La grotte de San Michele (Sisco, HauteCorse) : une sépulture originale de l'âge du Bronze corse, dans : Leduc M., Valdeyron N., Vaquer J. (Dir.), Sociétés et espaces. Actes des $\mathrm{III}^{\mathrm{e}}$ Rencontres Méridionales de Préhistoire Récente, (Toulouse, novembre 1998), Archives d'Ecologie Préhistorique, Toulouse, p. 253-256.

De Lanfranchi F.

1973: Les sépultures en grotte et en abri sous roche de l'âge du Fer, dans : L'âge du Fer en Méditerranée, Maison de la Culture de la Corse, Bastia, p. 105-118.

1978: Capula. Quatre millénaires de survivances et de traditions, Centre Archéologique de Lévie, 390 p.
1998: La torre de Tusiu (Altagène/ Altaghjè). Contribution à la connaissance des monuments circulaires de la Corse-du-Sud, dans : D'Anna A., BINDER D. (DIR.), Production et identité culturelle, Actes des IIes Rencontres Méridionales de Préhistoire récente (Arles, novembre 1996), APDCA, Antibes, p. 265-277.

Del Lucchese A., Maggi R.

1985: Considerazioni sulla cronologia dell'età del Bronzo in Liguria, Rivista di Studi Liguri, 48, p. 75-90.

Depalmas A.

2009: Il Bronzo finale della Sardegna, dans: Preistoria e Protostoria della Sardegna, Pre-Atti della XLIV Riunione Scientifica dell'Istituto Italiano di Preistoria e Protostoria (Cagliari, novembre 2009), Florence, p. 141-160.

Di Gennaro F.

1986: Forme di insediamento tra Tevere e Fiora dal Bronzo finale al principio dell'Età del Ferro, Studi Etruschi, 14, Florence, 211 p.

Domanico L.

1998 : Analisi degli indicatori cronologici delle necropoli del Bronzo finale in Etruria, dans : Negroni Catacchio N. (DIR.), Protovillanoviani elo protoetruschi, ricerche e scavi, Atti del Terzo Incontro di Studi dell'istituto "Preistoria e Protostoria dell'Etruria" (Farnese, mai 1995), Octavo, Milan, p. 53-78.

Germanì $\mathbf{F}$.

1999: Malattie e culture della Sardegna preistorica, dans : FLORIS G., SAnNa E. (DIR.), L'Uomo in Sardegna. Aspetti di Antropobiologia ed Ecologia umana, Sestu, p. 21-39.

Gilabert C., Leandri F.

2004: Le site mégalithique de Mamucci sur le plateau de Capo Castinco à Santo-Pietro-di-Tenda (Haute-Corse), dans : Dartevelle H. (Dir.), Auvergne et Midi. Actualité de la recherche, Actes des $\mathrm{V}^{\mathrm{e}}$ Rencontres Méridionales de Préhistoire Récente (Clermont-Ferrand, novembre 2002), Préhistoire du SudOuest, supplément n 9, p. 509-522.

Grosjean R., Liégeois J., Peretti G.

1976: Les civilisations de l'âge du Bronze en Corse, dans : Guilaine J. (DIR.), La Préhistoire Française, II, CNRS, Paris, p. 644-653. 
Leandri F., Tramoni P., Nebbia P., Pasquet A.

1996: Canton de Sartène, Bilan Scientifique de la région Corse, p. 29-31.

Leandri F., Gilabert C., Jorda C., Demouche $\mathbf{F}$.

2012: Monte Revincu (Santo-Pietro-diTenda, Haute-Corse) : aux origines du mégalithisme en Méditerranée, Errance, Paris, 132 p.

Magdeleine $\mathbf{J}$.

1995: Préhistoire du Cap Corse : les abris de Torre d'Aquila, Pietracorbara (Haute-Corse), Bulletin de la Société Préhistorique Française, XCII, p. 363-377.

MarchetTi M.-L.

2007 : Étude du site A Grotta Piatta (Aregno, Haute-Corse). Données nouvelles sur les pratiques funéraires protohistoriques de la Corse, dans : Tozzi C., WeIss M.C. (DIR.), Préhistoire et Protohistoire de l'aire tyrrhénienne Preistoria e Protostoria dell'area tirrenica, Felice, p. 57-65.

Milanini J.-L., David H., Pasquet A., Tramoni P.

2008: La sépulture de l'âge du Fer de Tappa 2 (Purtivecchju, Corse-du-Sud), Documents d'archéologie Méridionale, 31, p. 131-151.

Milletti M., Peche-Quilichini K., Amici S., Biancifiori E., Delvaux S., Lachenal T., Mottolese C., Pallone V., Palmieri S., Pretta G., Py V., Sartini E.

2012: Cuciurpula, Serra-di-Scopamena/ Sorbollano (Corse-du-Sud): nuovi dati su un insediamento protostorico corso (campagne 2008-2011), Materiali per Populonia, 9-10, p. 377-444.

Negroni Catacchio N.

1998: Proposta di una scansione cronologica del Bronzo finale nel territorio tra Fiora e Albegna, dans : Negron Catacchio N. (DiR.), Protovillanoviani elo protoetruschi, ricerche e scavi, Atti del Terzo Incontro di Studi dell' istituto "Preistoria e Protostoria dell'Etruria" (Farnese, mai 1995), Octavo, Milan, p. 79-97.

Pacciarelli M.

2001: Dal villaggio alla città. La svolta protourbana del 1000 a.C. nell'Italia tirrenica, Grandi Contesti e Problei della Protostoria Italiana, 4, 312 p.

Peche-Quilichini K.

2009: Fonds céramiques et vannerie dans le sud de la Corse au Bronze final, Bulletin de la Société Préhistorique Française, CVI, p. 569-580.

2010a : Le vase de fondation zoomorphe du premier âge du Fer de Cuciurpula (Serra-di-Scopamène/Sorbollano,
Corse-du-Sud), Bulletin de la Société Préhistorique Française, CVII, p. 371-381.

2010b: La ceramica del Bronzo finale nel sud della Corsica (sec. XII-IX): la facies "Apazzu-CastidettaCucuruzzu", dans : NEGRONI CATACCHIO N. (DIR.) - L'alba dell'Etruria. Fenomeni di trasformazione e di continuità nei secoli XII-VIII, Atti del Nono Incontro di studi del comitato "Preistoria e Protostoria dell'Etruria" (Valentano et Pitigliano, septembre 2008), p. 573-593.

2012a: De l'âge du Bronze à l'âge du Fer entre Ortolu et Rizzanese (Corse-duSud) « première partie » : céramique et chronologie, Etudes Corses, 74, p. 9-52.

2012b : Le Bronze final et le premier âge du Fer de la Corse : chronologie, production céramique et espaces culturels, Acta Archaeologica, 83, p. 203-223.

2012c: Dal Bronzo finale al primo Ferro nell'altro lato delle Bocche... Evidenze archeologiche di trasformazioni culturali in Corsica meridionale nei secoli XIII a VII a.C., dans : Preistoria e Protostoria della Sardegna, Atti della XLIV Riunione Scientifica dell'Istituto Italiano di Preistoria e Protostoria (Cagliari, novembre 2009), Florence, p. 1109-1114.

2013a: La collection céramique de Santa Barbara (Sartène, Corse-du-Sud) et la question de la transition Bronze final / Fer I dans le sud de la Corse, dans : Lo Schiavo F., Milletti M. (Dir.), Les lingots "peau de bouf » et la circulation en Méditerranée centrale jusqu'à l'époque archaïque, Actes du II ${ }^{e}$ colloque international de Mariana (Lucciana, septembre 2005), Piazzola, Ajaccio, p. 67-77.

2013b: De l'âge du Bronze à l'âge du Fer entre Ortolu et Rizzanese (Corse-duSud) « seconde partie »: hypothèses paléodémographiques et territoriales, Etudes Corses, 76, p. 9-37.

2014: Protohistoire d'une île. Vaisselles céramiques $d u$ Bronze final et du premier âge $d u$ Fer de Corse (1200-550 av. J.-C.), MontpellierLattes, 280 p. (Monographies d'Archéologie Méditerranéenne, 34).

À paraître: Ceci n'est pas (qu')une assiette. Ambiguïtés et ambivalences fonctionnelles des formes basses de la vaisselle de la fin de l'âge du Bronze de Corse, dans : Perrin T., Senepart I., Thirault E., Cauliez J. (Dir.) - « De la tombe au territoire ». Actualités de la recherche, Actes des $\mathrm{XI}^{\mathrm{e}}$ Rencontres Méridionales de Préhistoire Récente (Montpellier, septembre 2014).

Peche-Quilichini K., Bergerot L., lachenal T., Martinetti D., Py V., REgERT M.

2012: Les fouilles de Cuciurpula : la structure 1, dans : PeCheQuilichini K. (DIR.), L'âge du Fer en Corse - Acquis et perspectives, Actes de la table ronde de Serradi-Scopamène (août 2009), Associu Cuciurpula, Ajaccio, p. 35-57.

Peche-Quilichini K., Lachenal T., Pretta G.

2013: Nouvelles données sur l'habitat du premier âge du Fer en Alta Rocca, dans : De LanfranchiJ. (Dir.), Quoi de neuf en archéologie? Actes des $X I^{e}$ Rencontres du Musée de l'Alta Rocca (Levie, novembre 2011), Levie, p. 103-123.

Peche-Quilichini K., Delvaux S., Lachenal T., De Lanfranchi F.

2014a: Espaces de circulation, espaces de cheminement. Quelques «pistes» de réflexion pour le sud de la Corse entre Bronze final et premier âge du Fer, Bulletin de la Société Préhistorique Française, CXI, p. 101-128.

Peche-Quilichini K., Bec Drelon N., Biancifiori E., Boutoille L., Martin L., Mayca J., Rageot M., Recchia-Quiniou J. 2014b: L'habitation 6 de Cuciurpula (Serradi-Scopamena et Sorbollano, Corsedu-Sud). Eléments de définition chronologique, culturelle et économique du Bronze final 2-3 de Corse méridionale, dans : Perrin T., SÉnépart I., Leandri F., Cauliez J. (Dir.), Chronologie de la Préhistoire Récente dans le Sud de la France. Acquis 1992-2012. Actualités de la recherche. Actes des $X^{e}$ Rencontres Méridionales de Préhistoire Récente (Ajaccio, octobre 2012), AEP, Toulouse, 2014 p. 323-337.

Peche-Quilichini K., Lachenal T. Amici S., Bartoloni G., Bergerot L. Biancifiori E., Colomba Carraro E. Delvaux S., Grevey A.-L., De Lanfranchi F., Milletti M., Mottolese C., Volpi A.

2015: L'espace domestique au Bronze final et au premier âge du Fer dans le sud de la Corse, Trabajos de Prehistoria, 72, p. $259-281$

Peche-Quilichini K., De Lanfranchi F., Sourisseau J.-C., Py V.

À paraître 1 : Nuciaresa (Levie, Corsedu-Sud) et la fin du premier âge du Fer dans le sud de la Corse. Les fouilles de l'habitation 1 (VII $^{\mathrm{e}}-\mathrm{VI}^{\mathrm{c}}$ siècles av. J.-C.), Bulletin de la Société Préhistorique Française.

Recchia-Quiniou J., Peche-Quilichini K., Giannesini G.

À paraître : Vases anthropomorphes et zoomorphes du Néolithique et des âges des métaux en contexte méditerranéen. Études de cas, approche sociologique et degré de rémanence dans les sociétés traditionnelles sub-actuelles, dans : II Congreso Internacional de Estudios 
Cerámicos. Etnoarqueología y experimentación. Más allá de la analogía (Grenade, mars 2013).

Rittatore Vonwiller F.

1975: La cultura protovillanoviana, Popoli e Civiltà dell'Italia Antica, IV, Biblioteca di Storia Patria, Rome, p. 9-60.

\section{Rubat Borel F.}

Tra Protogolasecca e Gruppo RSFO : il gruppo Pont-Valperga e il Bronzo finale nel Piemonte nordoccidentale, dans : Vitali D. (Dir.) - Celtes et
Gaulois, l'archéologie face à l'histoire, 2, La Préhistoire des Celtes, Bibracte, 12, p. 197-202.

Soula F.

2012a: Organisation socio-territoriale des systèmes de pierres dressées dans l'aire corso-sarde : évolution chrono culturelle de deux exemples insulaires, dans : Perrin T., Senepart I., Cauliez J., Thirault E., Bonnardin S. (Dir.), Dynamismes et rythmes évolutifs des sociétés de la Préhistoire récente et actualité de la recherche, Actes des $I X^{e}$ Rencontres Méridionales de Préhistoire Récente (Saint-Georgesde-Didonne - Royan, Octobre 2010), AEP, p. 67-86.

2012b: Les pierres dressées de l'aire corso-sarde. Étude systémique des territoires, Thèse de doctorat, AixMarseille Université et Università degli Studi di Sassari, 1117 p.

Venturino Gambari M. (Dir.)

Navigando lungo l'Eridano. La necropoli protogolasecchiana di Morano sul Po, Museo Civico di Casale Monferrato, $222 \mathrm{p}$ 